\title{
Mathematical model of environmental waters purification
}

\author{
Yuri Skolubovich ${ }^{1 *}$, Alexey Skolubovich ${ }^{1}$, Dmitry Volkov $^{1}$, Tamara Krasnova ${ }^{2}$, and \\ Elena Gogina ${ }^{3}$ \\ ${ }^{1}$ Novosibirsk State University of Architecture and Civil Engineering (Sibstrin), Leningradskaya str., \\ 113, Novosibirsk, 630008, Russia \\ ${ }^{2}$ Kemerovo State University, Kemerovo, 630099, Russia \\ ${ }^{3}$ National Research Moscow State University of Civil Engineering, Yaroslavskoe shosse, 26, \\ Moscow, 129337, Russia
}

\begin{abstract}
This article describes the use of the stochastic approach, in particular, mass service theory and the development of its methods, adapted directly to the coagulation process as a mathematical tool. The coagulation process will be concerned as a) supplying water to the mixer, b) processing it with reagents (coagulants), c) settling for the mathematical analysis of water clarification effectiveness.
\end{abstract}

\section{Introduction}

One of the most effective physico-chemical methods for removing suspended colloidal particles from natural and wastewaters is coagulation, which allows achieving a high degree of water clarification [1]. The coagulation process will be concerned as a) supplying water to the mixer, b) processing it with reagents (coagulants), c) settling for the mathematical analysis of water clarification effectiveness.

We use the stochastic approach [2], in particular, mass service theory and the development of its methods, adapted directly to the coagulation process as a mathematical tool. Containing dispersed particles, the reagent enters the mixer simultaneously with water supply.

\section{Methods of experiment}

As the average time of a particle service, we mean the average time $t_{\mathrm{sev}}$, determined by the gap length, from the moment the reagent enters the mixer until flakes precipitation.

As incoming requirements we mean either particles that are already in the water and their average value (or concentration) which is already known, or entering into the mixer [3].

Formulation model. System $S$, consisting of storage and a service device, at random times receives requirements in accordance with the Poisson law with intensity $a$ :

\footnotetext{
*Corresponding author: michmacha@ mail.ru
} 


$$
V_{k}(t)=\frac{(\alpha t)^{k}}{k !} e^{-\alpha t}, k=0,1, \ldots .
$$

The storage capacity is limited and equal to $n$ requirements. If the incoming request finds the full storage, then it is lost. As soon as the service device is released (it finishes servicing the previous batch of requirements), it becomes the storage and accepts all the requirements there for servicing. [4] The service time of any requirement is random and distributed exponentially, with the parameter $\beta$

$$
F(t)=1-e^{-\beta t}, \beta=1 / t_{a t}
$$

$t_{a t}$ - average service time of the entire batch.

It is required to find $P_{k}(t)$ probability, at the moment of time $t \in[0, \infty) k$ is in the storage, requirements, $k=0,1,2, \ldots, n$.

The system of equations corresponding to the model is:

Normalization condition

$$
\left\{\begin{array}{l}
P_{0}^{\prime}(t)=-\alpha P_{0}(t)+\beta \sum_{k=1}^{n} P_{k}(t), \\
P_{k}^{\prime}(t)=-(\alpha+\beta) P_{k}(t)+\alpha P_{k-1}(t), 1 \leq k<n, \\
P_{n}^{\prime}(t)=-\beta P_{n}(t)+\alpha P_{n-1}(t) .
\end{array}\right.
$$

Initial conditions are defined as

$$
\sum_{k=0}^{n} P_{k}(t)=1
$$

In order to solve system (1), we note that

$$
P_{0}(0)=1, P_{k}(0)=0, k=1,2, \ldots, n .
$$

$$
\sum_{k=1}^{n} P_{k}(t)=1-P_{0}(t)
$$

then

$$
P_{0}^{\prime}(t)=-(\alpha+\beta) P_{0}(t)+\beta
$$

Given the initial conditions, the solution of this linear equation is

if $k=1$, we have

$$
P_{0}(t)=\frac{\beta}{\alpha+\beta}+\frac{\alpha}{\alpha+\beta} e^{-(\alpha+\beta) t} .
$$

Substituting instead $P_{0}(t)$ its expression (3), we get

$$
P_{1}^{\prime}(t)=-(\alpha+\beta) P_{1}(t)+\alpha P_{0}(t) .
$$

$$
P_{1}^{\prime}(t)=-(\alpha+\beta) P_{1}(t)+\frac{\alpha \beta}{\alpha+\beta}+\frac{\alpha^{2}}{\alpha+\beta} e^{-(\alpha+\beta) t} .
$$

This is a linear equation can be solved, like the previous one, we get

$$
P_{1}(t)=\frac{\alpha \beta}{(\alpha+\beta)^{2}}-\frac{\alpha \beta}{(\alpha+\beta)^{2}} e^{-(\alpha+\beta) t}+\frac{\alpha^{2} \cdot t}{\alpha+\beta} e^{-(\alpha+\beta) t}
$$


Continuing the process of solving the equations of system (1) recurrently, we obtain for

$$
k \leq n-1 P_{k}(t)=\frac{\alpha^{k} \beta}{(\alpha+\beta)^{k+1}}\left(1-e^{-(\alpha+\beta) t}\right)+e^{-(\alpha+\beta) t} \cdot\left[\frac{\alpha^{k+1} t^{k}}{k !(\alpha+\beta)}-\alpha^{k} \beta \cdot \sum_{s=1}^{k-1} \frac{t^{s}}{s !(\alpha+\beta)^{k-s+1}}\right] \text {. }
$$

If $k=n$, we have a linear equation

$$
P_{n}^{\prime}(t)=-\beta P_{n}(t)+\alpha P_{n-1}(t)
$$

Its overall solution is

$$
P_{n}(t)=e^{-\beta t} \cdot \int_{0}^{t} \alpha P_{n-1}(x) d x, \text { где } P_{n-1}(0)=0 .
$$

You can also use the normalization condition (2), then

$$
P_{n}(t)=1-\sum_{k=0}^{n-1} P_{k}(t)
$$

As we see, the formulas for the transitional regime are hard to predict and unsuitable for practical use.

However, they are greatly simplified for the stationary mode that takes place in practice,.

In fact, we have

$$
\forall k, \quad \lim _{t \rightarrow \infty} P_{k}(t)=p_{k}>0
$$

then

$$
\left\{\begin{array}{l}
p_{k}=\frac{\alpha^{k} \beta}{(\alpha+\beta)^{k+1}}, k=0,1, \ldots, n-1, \\
p_{n}=\left(\frac{\alpha}{\alpha+\beta}\right)^{n} .
\end{array}\right.
$$

Considering formulas (6), we introduce and calculate the following quality indicators:

$P_{\mathrm{d} .}-$ the likelihood that the request will be denied service,

$N$ - average number of requests in the drive,

$$
N=\sum_{k=1}^{n} k P_{k}
$$

$K$ - drive payload ratio,

$$
K=\frac{N}{n}
$$


$M$ - the reliability of the required storage capacity is equal to $\gamma$,

where $P_{\mathrm{d}}=1-\gamma, \beta$ - storage performance, $\alpha$-incoming requirements.

We estimate the error from hypothesis that the process is stationary.

The absolute error is determined from the formula

$$
A_{k}=P_{k}(t)-p_{k}
$$

The relative error will be equal to

$$
\Delta A_{k}=\left(P_{k}(t)-p_{k}\right) / p_{k}, k=0,1, \ldots, n-1 .
$$

a) absolute

$$
A_{\text {отК }}=P_{k}(t)-p_{n}
$$

б) relative

$$
\Delta A_{\text {отК }}=\left(P_{k}(t)-p_{n}\right) / p_{n} .
$$

Suppose, given in $\mathrm{m}^{3}$, the volume of wash water contains, on average, $\alpha=0,02 \%$ of colloid-dispersed particles, and the coagulants are able to effectively process per hour, on average, $\beta=0,025 \%$ of these particles contained in the mixer with volume $\mathrm{n}=2$ units of volume (per unit volume take $50 \mathrm{~m}^{3}$ ). The reliability of the required volume of the mixer $\gamma=$ 0,95 . [5]

Calculating the quality indicators, we have

1. probability that the drive is full:

2. the average volume of wash water contained in the drive

3. payload ratio

$$
N=1 P_{1}+2 P_{2}=\frac{\beta \alpha}{(\alpha+\beta)^{2}}+\frac{2 \alpha^{2}}{(\alpha+\beta)^{2}} \approx 0,642\left(m^{3}\right)
$$

4. required average size of the drive

$$
K=\frac{N}{n}=\frac{0,642}{2}=0,32(32 \%) .
$$

$$
M=\frac{\ln P_{\text {отк }}}{\ln \alpha-\ln (\alpha+\beta)}=\frac{\ln 0,198}{\ln 0,02-\ln 0,045} \approx 1,997
$$

Calculate the variance

$$
\begin{aligned}
D=1 \cdot p_{1}+2^{2} \cdot p_{2}-N^{2} & =\frac{\beta \cdot \alpha}{(\alpha+\beta)^{2}}+\frac{4 \cdot \alpha^{2}}{(\alpha+\beta)^{2}}-N^{2}=\frac{0,02 \cdot 0,025}{(0,02+0,025)^{2}}+ \\
& +\frac{4 \cdot(0,02)^{2}}{(0,02+0,025)^{2}} \approx 0,6248
\end{aligned}
$$

For the standard deviation we get $\sigma=\sqrt{0,6248} \approx 0,79$.

With reliability $\gamma=0,95 \mathrm{t}$ is enough to take a deviation equal to one $\sigma$, i.e. mixer volume $V$ must be no less than

$$
V=M+\sigma=1,997+0,79 \approx 2,78 \text {. }
$$


Or absolute units $V=2,78 \cdot 50=139\left(\mathrm{~m}^{3}\right)$.

The figure shows the system curves entering the stationary mode for probabilities $P_{k}(t), k=0,1,2$, calculated by formulas (3) - (5), for the transient mode, and by formula (b), for the stationary mode, with $n=2, \alpha=0,02, \beta=0,025$.

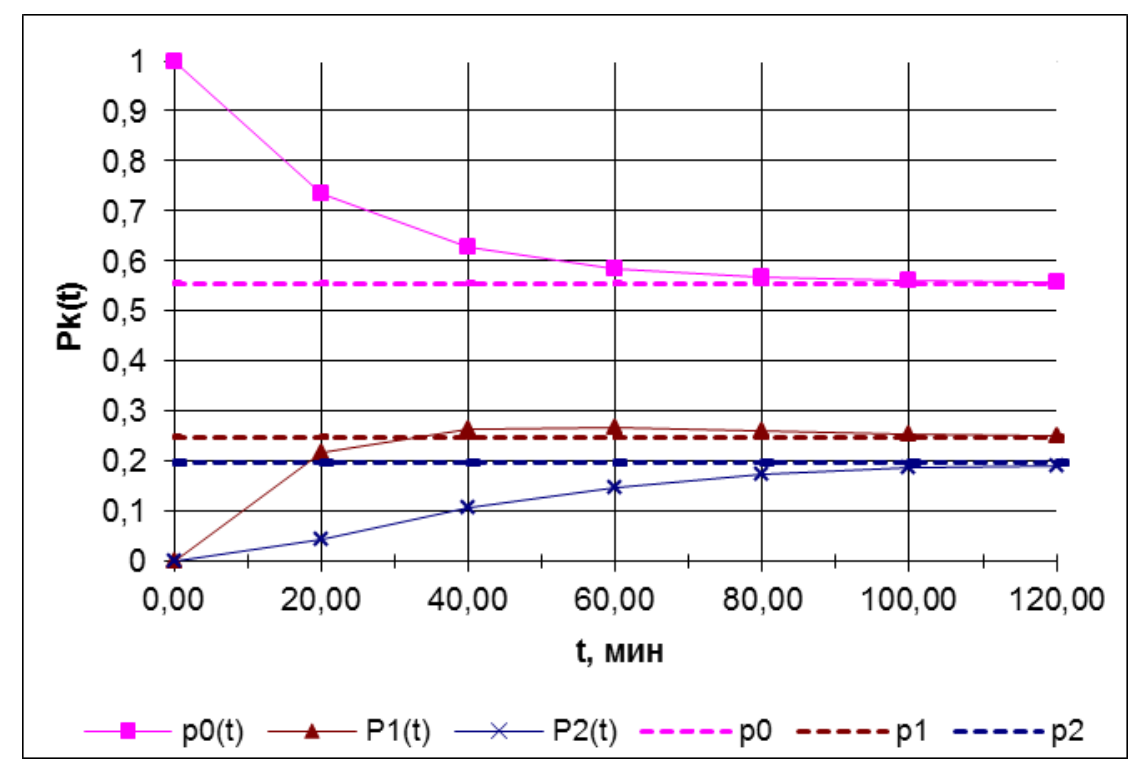

Fig. 1. Probabilities $P_{k}(t), k=0,1,2$, of the system state $S$ with $n=2, \alpha=0,02, \beta=0,025$.

For comparison, the horizontal dashed lines are the values of the corresponding probabilities $p_{k}$ for the stationary process $[6,7,8]$.

\section{Conclusion}

It can be seen from the figure that the system quickly enters the stationary mode of operation and already at $\mathrm{t}>30$ minutes, you can use formulas (b) [9].

Thus, queuing theory models allow not only analyzing the coagulation process as a whole, but also effectively planning the water purification process [10].

\section{References}

1. A.M. Blagorazumova, Treatment and dewatering of urban wastewater sludge (Lan. P., 2014).

2. T.I. Khalturina, O.B. Churbakova, Construction. 11-12, 84-89 (2012).

3. Yu.L. Skolubovich, Preparation of drinking water from underground sources (NGASU, Sibstrin, 2014).

4. V.B. Smirnov, D.A. Guskov, Highly effective granular filters for post-treatment of biologically treated sewage. Plumbing, heating, air conditioning. 6 (2017).

5. E.L. Voytov, Y.L. Scolubovich, Preparation of drinking water from surface sources with a high natural and anthropogenic pollution (NSUACE, Sibstrin, 2010). 
6. E.L. Voitov, Y.L. Skolubovich, Cleaning Water device. Pat. 2307075 RF, MKI C 02 F 1/52. Bul. 27 [5] (2015).

7. T. Wik, On modeling the dynamics of fixed biofilm reactors (Chalmers University of Technology, 2013).

8. V.B. Smirnov, D.A. Guskov, Highly effective granular filters for post-treatment of biologically treated sewage. Plumbing, heating, air conditioning. 6 (2017).

9. C.F. Forster, D A. J. Wase, Environmental biotechnology (Chichester, 2014).

10. Yu.V. Voronov, S.V. Yakovlev, Wastewater and wastewater treatment (Publishing Association of Construction Universities, 2010). 